\title{
Resting metabolic connectivity in Alzheimer's disease
}

\author{
Silvia Morbelli • Dario Arnaldi - Selene Capitanio • \\ Agnese Picco $\cdot$ Ambra Buschiazzo · Flavio Nobili
}

Received: 22 May 2013/Accepted: 27 June 2013/Published online: 16 July 2013

(C) Italian Association of Nuclear Medicine and Molecular Imaging 2013

\begin{abstract}
Metabolic connectivity analysis of resting ${ }^{18} \mathrm{~F}$-FDG PET is based on the assumption that brain regions whose metabolism is significantly correlated at rest are functionally associated and that the strength of the association is proportional to the magnitude of the correlation coefficient. Therefore, this method could be used to evaluate connectivity networks independently on the basis of performance in specific tasks. Published studies have provided evidence that metabolic connectivity substantially overlaps underlying anatomical pathways and depends on the location of the analyzed regions, but is not influenced by their size. The present review focuses on the methods and meaning of resting inter-regional correlation analysis of cerebral metabolic rate of glucose consumption in Alzheimer's disease. Accordingly, we describe the evolution of analytical tools from the correlation with a single region of interest to a voxel-based statistical parametric mappingbased approach. We also discuss the pathophysiological implications of metabolic connectivity studies both for Alzheimer-related disconnection syndrome and for default-mode network impairment and compensation mechanisms.
\end{abstract}

S. Morbelli · S. Capitanio - A. Buschiazzo

Nuclear Medicine Unit, Department of Health Sciences, IRCCS AOU San Martino-IST, University of Genoa, Largo R. Benzi 10, 16132 Genoa, Italy

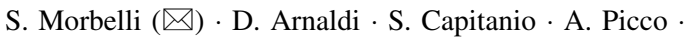

A. Buschiazzo · F. Nobili

Clinical Neurophysiology, Department of Neurosciences, Ophthalmology and Genetics, IRCCS AOU San Martino-IST, University of Genoa, Largo R. Benzi 10, 16132 Genoa, Italy

e-mail: silviadaniela.morbelli@hsanmartino.it
Keywords Metabolic connectivity - Alzheimer's disease · Brain 18F-FDG PET · Default-mode network

\section{Introduction}

Alzheimer's disease (AD) is the most prevalent dementing disorder worldwide and is pathologically defined by the presence of amyloid aggregations (neuritic plaques, NP) and tau pathology (neurofibrillary tangles, NFT) [1, 2]. Neuropathological data in AD reveal that NFT are prominent in the medial temporal lobe (MTL) early in the disease and then progress outward [3], while NP have a broader cortical distribution that includes but is not especially prominent in the MTL [4]. Moreover, recent pathological studies have led to the hypothesis that NFT predominate in pyramidal neurons that form corticocortical connections between and within the cerebral hemispheres, whereas NP are prevalent at the end of these tracts and in their collateral branches [5, 6]. Consequently, AD pathophysiology could be the result not only of damage to one or more neuronal systems, but also of a disruption of the brain's connectivity due to abnormal interactions between neuronal systems [6].

Consistent with this pathology, magnetic resonance imaging (MRI) in early stage AD demonstrates MTL atrophy [7, 8] while functional imaging with ${ }^{18}$ F-FDG PET reveals hypometabolism with a characteristic parietotemporal and posterior cingulate pattern [9].

The concept of a corticocortical disconnection syndrome in $\mathrm{AD}$ was initially investigated using blood-oxygen-leveldependent (BOLD) fMRI, which can identify functional networks related to the joint activation of brain areas involved in different cognitive functions [10]. Several fMRI studies have shown that patterns of activation are changed in $\mathrm{AD}$ patients during the performance of certain 
tasks [10, 11]. Similarly, PET activation studies with $\mathrm{H}_{2}^{15} \mathrm{O}$ have also been analyzed in terms of functional connectivity and have demonstrated reduced functional interactions implying an anterior-posterior disconnection [12]. Since these approaches focus on the networks that directly underlie specific task performance, they do not evaluate the more general concept of impaired connectivity in $A D$, nor do they directly address impairment of the default-mode network (DMN). This network, which includes the medial prefrontal cortex, posterior cingulate, and inferior parietal lobule, is thought to be mainly active in resting conditions when individuals are engaged in internally focused tasks including autobiographical memory retrieval, visualizing the future, and conceiving the perspectives of others [13]. Several lines of evidence suggest that in the preclinical stage of $\mathrm{AD}$, beta amyloid deposition is present mainly in DMN regions [14] and that impairment of the DMN may represent a sensitive and specific biomarker of incipient AD. Among the methods proposed for studying the DMN in $\mathrm{AD}$, resting-state fMRI reflects spontaneous neuronal activity and/or the endogenous neurophysiological processes of the human brain in the resting state (see Liu et al. for a review [15]), while $\mathrm{H}_{2}^{15} \mathrm{O}$ PET studies allow analysis of covariance of rest-specific regional cerebral blood flow patterns [16]. Recent combined resting-state fMRI and amyloid PET studies have provided, in vivo, further evidence of the pathophysiological meaning of impaired connectivity in AD. Indeed, two elegant studies, by Drzezga et al. [14] and Sheline et al. [17], investigated disruptions of functional whole-brain connectivity in nondemented older individuals with increased amyloid burden and demonstrated a negative correlation between wholebrain connectivity values and amyloid burden. These results indicate that not only hypometabolism but also disruption of connectivity may be an early functional consequence of emerging molecular pathology of $\mathrm{AD}$ evolving prior to the clinical onset of dementia. Finally, resting-state functional connectivity and the default network can be investigated using ${ }^{18} \mathrm{~F}$-FDG PET measures of resting glucose metabolism independently of vascular coupling [18-22]. The present review focuses on the methodology and meaning of this latter analysis, which is more often referred to as metabolic connectivity analysis.

\section{The rationale of metabolic connectivity analysis}

Among the functional neuroimaging techniques, ${ }^{18} \mathrm{~F}-\mathrm{FDG}$ PET has the unique ability to estimate the local cerebral metabolic rate of glucose consumption (CMRgl), thus providing information on the distribution of neuronal death and synapse dysfunction in vivo [23]. ${ }^{18} \mathrm{~F}-\mathrm{FDG}$ is transported into in the cell, just like glucose, and then trapped.
As a result, it has become a well-established in vivo marker for measuring the CMRgl [24, 25]. Indeed, the high energy demands of neuronal synaptic activity are met by the provision of lactate through glia cells, leading to increased glucose consumption in these cells [26]. In particular, when

${ }^{18} \mathrm{~F}$-FDG PET is performed at rest, glucose uptake distribution is driven mainly by basal neuronal glutamatergic activity and, therefore, provides an index of general neural integrity [27]. Thus, glucose metabolism has been shown to be closely coupled to neuronal function both at rest and during functional activation [26]. In the field of degenerative dementias, specifically that of AD, FDG PET plays a major clinical role by showing (or not) a disease-specific pattern of hypometabolism thought to correspond broadly to the brain regions damaged in the earliest stages of the disease [28], which are, interestingly, part of the DMN. However, ${ }^{18} \mathrm{~F}$-FDG studies both in patients with neurodegenerative diseases [29] and in patients with brain tumors $[27,30]$ also revealed hypometabolism in brain areas neurally connected to the structurally damaged site. This phenomenon, called diaschisis, is well evident also in the clinical single-patient setting and implies that ${ }^{18} \mathrm{~F}-\mathrm{FDG}$ PET is capable of identifying functional alterations determined by the loss of neuronal connections.

Accordingly, by calculating correlation coefficients-or patterns of intercorrelations-between FDG uptake values, it is possible to estimate the functional association between cerebral areas.

Investigation of intercorrelation patterns of FDG uptake [18] provided results substantially overlapping with anatomo-functional data, thus legitimizing the assertion that functional interactions reflect the underlying anatomical pathways. Therefore, interregional correlations of glucose metabolic rates are generally discussed in terms of "traffic (metabolic-functional connectivity) on the anatomical 'roads' present in the brain" which, by contrast, can be investigated using other neuroimaging methods, such as diffusion tensor imaging [21, 31].

\section{Resting metabolic connectivity: evolution of the method}

The first studies investigating resting intercorrelations of glucose metabolic rates between brain regions in $\mathrm{AD}$ were published in the 80 s by Horwitz and colleagues [19]. These authors defined functional interactions as the intercorrelations between different brain regions. The basic concepts are that pairs of brain regions whose regional CMRgl (rCMRgl) values are significantly correlated are functionally associated and that the strength of the association is proportional to the magnitude of the correlation coefficient.

This study [19] evaluated rCMRgl in 28 pairs of bilateral and three midline regions of interest (ROI) in $\mathrm{AD}$ 
patients and controls; then, for both AD patients and controls, the rCMRgl measurements were used in separate correlation matrices to evaluate partial correlation coefficients between the ROIs. In these correlation matrices, the brain regions were divided into frontal, parietal, temporal, occipital, and non-cortical seeds. A reduced number of frontal-parietal correlations and a loss of partial correlations between homologous right-left ROI were highlighted in AD patients with respect to controls. Moeller and colleagues [32] approached the study of the brain metabolic network in more formal mathematical-statistical terms, through the Scaled Subprofile Model (SSM). This novel model, basically a form of principal component analysis, explicitly represented rCMRgl profiles as a combination of region-independent global effects, a group mean pattern, and a mosaic of interacting networks. They applied this approach in 12 demented AIDS patients and 18 normal controls. Two significant metabolic covariance pattern descriptors that together accounted for $71-96 \%$ of the rCMRgl variation across subjects for 22/28 regions in the AIDS group were extracted. These descriptors were highly correlated with performance on several neuropsychological tests, providing independent validation of the analysis technique as a means of discovering and describing behaviorally related components of group rCMRgl profiles.

In the early 90s, Azari and colleagues [33] applied a similar multiple regression/discriminant analysis to assess individual differences in patterns of rCMRgl interdependencies and confirmed weaker functional interactions (less positive correlations) in $\mathrm{AD}$ patients. In the wake of these pioneering studies, the concept of metabolic connectivity was proposed, based on the same assumptions but exploiting the statistical parametric mapping (SPM) approach. Mosconi et al. [20] performed whole-brain voxel-based correlation analyses to assess functional interactions of the entorhinal cortex (EC), defined as voxels, whose activity significantly correlates with rCMRgl in the EC. EC rCMRgl data were extracted from the left and right hemispheres by means of the Marsbar toolbox for both controls and AD patients. Then, EC rCMRgl values were used as covariates of interest in a further SPM analysis. More recently this SPM approach to metabolic connectivity has been refined by Lee et al. [21], who systematically explored metabolic connectivity based on voxel-wise interregional correlation analysis of SPM in normal healthy adults, thus establishing normative data of interregional metabolic connectivity. In this study, PET images were spatially normalized to the Korean standard PET template of young male adult subjects; then, FDG mean counts were extracted using a structural probabilistic map for 70 brain volumetric regions (volumes of interest, VOI) $[34,35]$ and 28 cytoarchitectonically defined VOI [36]. Probability-weighted mean counts for each seed VOI were globally normalized with respect to individual gray matter mean counts.

For interregional correlation analysis, extracted mean regional VOI counts were used as covariates to find regions showing significant voxel-wise correlations across subjects using SPM2. Moreover, to evaluate the effect of VOI size on correlation extent, seed VOI sizes were compared with sizes and size ratios, respectively, of correlated areas. This study confirmed that interregional correlation analysis of FDG PET maps offers a means of examining voxel-wise regional metabolic connectivity of the resting human brain. Moreover, the findings of Lee et al. indicate that patterns of metabolic connectivity of functional brain units depend on their regional locations and are not influenced by VOI size. Finally, an alternative way of assessing metabolic connectivity has been provided by Huang and colleagues [37] who proposed a method based on sparse inverse covariance estimation (SICE) for identifying functional brain connectivity networks from ${ }^{18} \mathrm{~F}$-FDG PET data. After preprocessing of the images, the authors built a brain connectivity model for $\mathrm{AD}$ by computing a sample covariance matrix of 42 VOI (in the frontal, parietal, occipital, and temporal lobes) in $49 \mathrm{AD}$ patients. This method was able to identify both the structure and strength of the connectivity network for a large number of brain regions, even with small sample sizes. The authors suggested that this method can also be used for classifying new subjects, which would make it a suitable approach for novel connectivity-based AD biomarker identification.

\section{Metabolic connectivity and the hippocampal issue}

The EC and the hippocampus are among the first regions targeted by AD pathology [38]. However, while several observations suggest that early hippocampal structural brain damage in $\mathrm{AD}$ is coupled with hypofunction and specifically reduced brain glucose metabolism, hippocampal hypometabolism is not always evident on PET studies [38]. Conversely, hypometabolism can be found in the posterior parieto-temporal cortex, lateral occipital cortex, precuneus, and posterior cingulate (PC) $[9,29]$ in various combinations in individual cases. It has thus been hypothesized that decreased posterior parietal and PC activity in prodromal $\mathrm{AD}$ (pAD) reflects decreased connectivity especially with the EC and hippocampus [39]. This hypothesis, which fits well with the concept that ${ }^{18} \mathrm{~F}$ FDG PET reflects brain glucose consumption at synaptic level, has been tested and confirmed by two metabolic connectivity studies. In the already mentioned study by Mosconi et al. [20] that a voxel-based correlation analysis of EC indicated an altered functional relationship between the EC and several cortical and limbic regions in $\mathrm{AD}$ with 
respect to normal aging. These results were confirmed using a different approach to metabolic connectivity analysis. Indeed, other authors [37], by means of SICE analysis, demonstrated that compared with normal controls, AD patients show a decrease in the amount of interregional functional connectivity within the temporal lobe, especially between the areas around the hippocampus. Similarly, more recently, in the context of a project by the European Alzheimer's Disease Consortium (EADC) [22], a metabolic connectivity analysis of both hippocampi was performed in 36 pAD patients and in two independent groups of 36 and 69 age-matched healthy controls. Prior to interregional correlation analysis, the area of hypometabolism in pAD was assessed. This preliminary step aimed to identify regions characterized, or otherwise, by relative hypometabolism in $\mathrm{AD}$ with respect to controls. As compared with the controls, the pAD patients showed relative hypometabolism in a large region including the posterior parietal cortex, precuneus, PC in both hemispheres, and lateral temporal lobe in the left hemisphere. Therefore, like previous PET studies, this analysis failed to find significant hippocampal hypometabolism in $\mathrm{AD}$ in comparison with controls. Accordingly, the authors specifically aimed to identify impairment of hippocampal metabolic connectivity even in the absence of hippocampal hypometabolism. In both the patient and control groups, extracted normalized mean hippocampal VOI counts were used as covariates to find regions showing significant voxel-wise correlations across subjects according to the procedure validated by Lee et al. [21]. Besides the expected autocorrelation, in both control groups, hippocampal metabolism showed correlations with the temporal, frontal, occipital cortex, and caudate nucleus. By contrast, in pAD patients, metabolism of both hippocampi showed autocorrelation and correlation with both the contralateral one and the amygdala. These findings strongly support the hypothesis that decreased posterior cingulate cortex activity in $\mathrm{pAD}$ reflects decreased connectivity, especially with the EC and hippocampus, and indirectly testifies to their damage. Figure 1 shows an example of metabolic connectivity analysis of the left hippocampus in controls and in a group of $\mathrm{pAD}$ patients.

\section{Metabolic connectivity and the default-mode network}

In addition to this confirmation of hippocampal disconnection, Morbelli et al. [22] provided further insight into DMN alteration in AD. In fact, both hippocampi showed correlations with the cuneus, precuneus, and posterior cingulate in controls, but not in pAD patients. Several fMRI studies of resting-state functional connectivity suggested that the hippocampus, even when not engaged in a specific task, is functionally connected with the DMN, in particular with the posterior cingulate and precuneus [40]. The lack of DMN-hippocampal metabolic correlation in the pAD group is thus in keeping with the reported perturbation of the DMN in AD [40] and is consistent with the expected abnormalities in large-scale memory networks [41]. In another study by the multicenter EADC group, metabolic connectivity analysis was used to investigate the presence of cognitive reserve (CR) in the early stages of $\mathrm{AD}$ [42]. The results of this study are discussed in detail in the next paragraph. However, as well as confirming the existence of $\mathrm{CR}$ in $\mathrm{AD}$ patients, the results of this second study provide further support for the importance of the DMN in AD pathophysiology. In fact, evidence from this study proved that $\mathrm{CR}$ in pAD patients is mediated by a prefrontal-posterior network which partially overlaps the topography of the DMN. The DMN is thought to be deactivated during task performance and this deactivation has been shown to be less pronounced in the early stage of AD. Previous studies demonstrated that CR modulates not only task-related brain activation areas but also deactivations of the DMN [43]. Since the degree of deactivation of the DMN is thought to reflect cognitive effort in elders [43], the pattern of reduced deactivation in the DMN in high CR elders has been interpreted in terms of more automatic processing [44]. In keeping with this hypothesis, the metabolic network highlighted by Morbelli et al. [42] was found to be more pronounced in highly educated pAD, even with respect to highly educated controls.

\section{Metabolic connectivity and cognitive reserve}

Cognitive reserve is the term used to refer to the hypothesized capacity of an adult brain to cope with brain damage in order to minimize symptomatology [45]. In the field of neuroimaging, CR has been defined by the difference between an individual's expected cognitive performance, given a particular level of imaging-assessed brain damage, and his actual cognitive performance.

Several ${ }^{18}$ F-FDG PET studies have demonstrated the presence of $\mathrm{CR}$ in $\mathrm{AD}$ patients, albeit mainly indirectly, through the demonstration of an inverse relationship between metabolism $\left({ }^{18} \mathrm{~F}-\mathrm{FDG}\right)$ and education/occupation [46] or intellectual ability [47]. To better understand CR, it is, however, crucial to identify the networks (not only regions) that allow implementation of $\mathrm{CR}$ in the presence of AD-related damage, and cognitive activation studies using $\mathrm{H}_{2} \mathrm{O}^{15}$ PET and fMRI have been performed [48] to pursue this objective. However, it can be hypothesized that CR is mediated by a generic network that is not necessarily taskspecific. Accordingly, metabolic connectivity analysis could be an ideal tool for testing the hypothesis of the presence of a CR-specific network in $\mathrm{AD}$. In the 
Fig. 1 Example of the results of voxel-wise interregional correlation analysis of the left hippocampus (HC) in 36 normal elderly control subjects (CTR) (a) and 36 prodromal Alzheimer's disease patients (pAD) (b). In the CTR, left HC metabolism showed, besides the expected autocorrelation, correlations with the temporal, frontal, and occipital cortex and striatal nucleus bilaterally, while in the pAD, HC metabolism showed only autocorrelation and correlation with contralateral homologous $\mathrm{HC}$

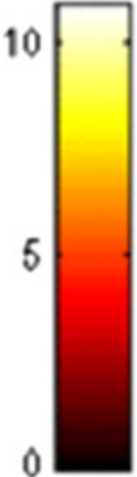

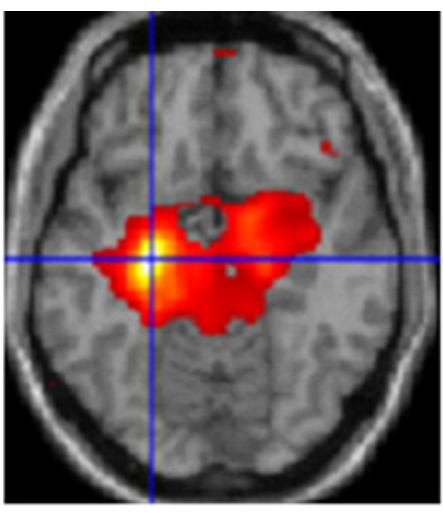

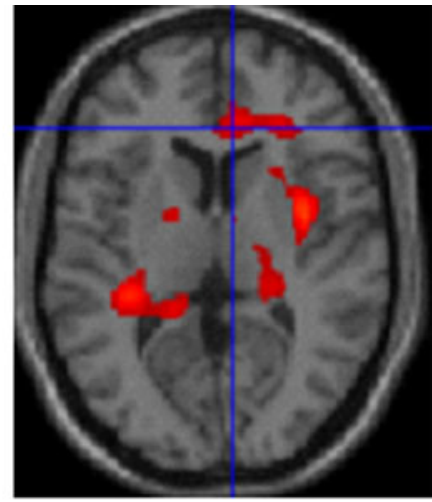

B

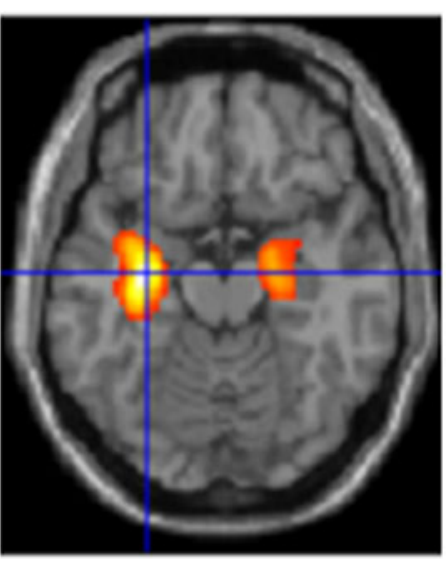

aforementioned EADC study [42], several preliminary steps were taken prior to the metabolic connectivity analysis. In particular, to investigate functional mechanisms that mediate $\mathrm{CR}$, highly and poorly educated $\mathrm{pAD}$ patients expressing the same level of cognitive symptoms were compared bidirectionally. On the one hand, this comparison provided confirmation that AD-typical damage is more pronounced in highly educated subjects. On the other, the analysis demonstrated the presence of relatively higher metabolic levels in the right inferior, middle, and superior frontal gyri in highly educated pAD. These regions, substantially corresponding to the right dorsolateral prefrontal cortex (DLFC), were then saved as VOI, normalized to cerebellar counts and included as covariates in the subsequent metabolic connectivity analysis not only in highly and poorly educated pAD patients, but also in two groups of highly and poorly educated age-matched controls. The importance of performing this analysis both in patients and in controls is that it makes it possible to investigate both the neural reserve and neural compensation components of CR. In fact, according to the definition of Yaakov Stern, neural reserve reflects preexisting brain networks that are more efficient or have greater capacity and may, therefore, be less susceptible to disruption, while neural compensation refers to the adoption of new, compensatory brain networks after pathology has impacted on those networks typically utilized for particular tasks [49]. In highly educated pAD patients, metabolism in the DLFC correlated extensively with that of several cortical areas in both hemispheres (frontal, temporal, occipital cortex, parahippocampal gyrus, and precuneus) as well as with that of the cerebellar hemispheres, while it was basically only autocorrelated in poorly educated pAD. When metabolic connectivity analysis of the right DLFC was extended to the control groups, it was demonstrated that the extensive DLFC-related metabolic network highlighted in highly educated pAD was indeed present and topographically similar in highly educated controls, but was quantitatively less pronounced with respect to what was observed in highly educated pAD. Accordingly the connectivity analysis demonstrated that $\mathrm{CR}$ in $\mathrm{pAD}$ patients with a high level of education is a result of both neural reserve and neural compensation. See Fig. 2 for a point-by-point comparison of the distribution of the results of the voxel-wise interregional correlation analysis of the DLFC in a group of a highly educated pAD patients and in a group of age- and education-matched controls.

\section{Metabolic connectivity as a diagnostic probe?}

An interesting possibility, to be exploited and explored in further research, is that of using resting metabolic 
Fig. 2 Point-by-point comparison of the distribution of the results of the voxel-wise interregional correlation analysis of the dorsolateral prefrontal cortex (DLFC) in a group of highly educated pAD patients and a group of age- and education-matched controls. Brain regions significantly correlated with DLFC metabolism are superimposed on an MRI scan and shown in green for $\mathrm{pAD}$ and in red for controls. The extensive DLFCrelated metabolic network highlighted in highly educated $\mathrm{pAD}$ is present and topographically similar in highly educated controls but is less extensive with respect to what is observed in highly educated $\mathrm{pAD}$
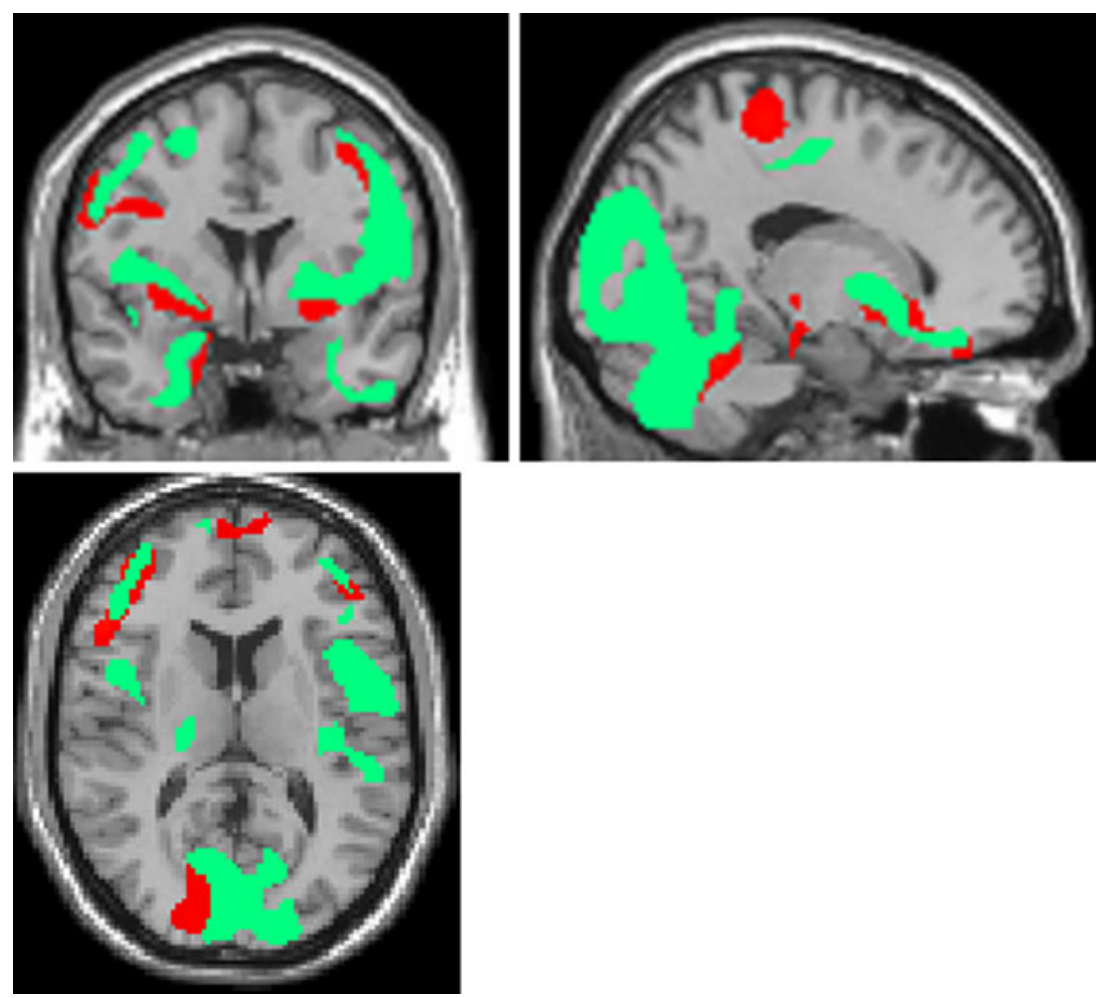

connectivity analysis on an individual basis to predict cognitive decline or response to disease-modifying therapy. To this end, Toussaint and colleagues [50] investigated the diagnostic power of FDG metabolic patterns in AD by combining voxel-based group analysis and independent component analysis to extract differential characteristic patterns from ${ }^{18} \mathrm{~F}$-FDG PET data in a large cohort of normal elderly controls and patients with AD. The obtained patterns were then used in conjunction with a support vector machine to discriminate $\mathrm{pAD}$ patients within a group of subjects with mild cognitive impairment (MCI). This approach showed $80 \%$ accuracy ( $85 \%$ sensitivity and $75 \%$ specificity) and thus allowed improved and early detection and differentiation of typical versus pathological metabolic patterns in the MCI population. The authors concluded that this method has the potential to identify, early in the course of the disease, individuals at high risk of rapid cognitive decline who could be candidates for new therapeutic approaches. Although these results are very intriguing, further studies and, possibly, userfriendly software development are still needed to establish whether this approach could be used by the pharmaceutical industry to better stratify subjects in secondary prevention trials or for clinical diagnostic purposes.

\section{Conclusions}

Metabolic connectivity analysis has provided new insight into $\mathrm{AD}$ pathophysiology by providing further evidence of
DMN impairment, hippocampal disconnection, and both cognitive reserve and compensation mechanisms in the preclinical stage of the disease. Metabolic connectivity studies show that supplementing standard inspection with a comprehensive evaluation of ${ }^{18} \mathrm{~F}-\mathrm{FDG}$ distribution in the resting brain may allow a more complete understanding of $\mathrm{AD}$ pathophysiology and possibly increase the diagnostic sensitivity of ${ }^{18}$ F-FDG PET. Further studies are needed to evaluate whether resting metabolic connectivity studies can be used on an individual basis to predict cognitive decline or response to disease-modifying therapy.

Conflict of interest S. Morbelli, D. Arnaldi, S. Capitanio, A. Picco, A. Buschiazzo, F. Nobili declare that they have no conflict of interest.

Human and Animal Studies This article does not contain any studies with human or animal subjects performed by any of the authors.

\section{References}

1. National Institute on Aging (1997) Consensus recommendations for the postmortem diagnosis of Alzheimer's disease. The National Institute on Aging, and Reagan Institute Working Group on Diagnostic criteria for the Neuropathological Assessment of Alzheimer's disease. Neurobiol Aging 18:S1-S2

2. Buckner RL, Snyder AZ, Shannon BJ et al (2005) Molecular, structural, and functional characterization of Alzheimer's disease: evidence for a relationship between default activity, amyloid, and memory. J Neurosci 25:7709-7717 
3. Pearson RC, Esiri MM, Hiorns RW, Wilcock GK, Powell TP (1985) Anatomical correlates of the distribution of the pathological changes in the neocortex in Alzheimer's disease. Proc Natl Acad Sci USA 82:4531-4534

4. Braak H, Braak E (1991) Neuropathological stageing of Alzheimer-related changes. Acta Neuropathol 82:239-259

5. Morrison J, Scherr S, Lewis D et al (1986) The laminar and regional distribution of neocortical somatostatin and neuritic plaques: implications for Alzheimer's disease as a global neocortical disconnection syndrome. In: Scheibel A, Wechsler A, Brazier M (eds) The biological substrates of Alzheimer's disease. Academic Press, Orlando, pp 115-131

6. Delbeuck X, Van der Linden M, Collette F (2003) Alzheimer's disease as a disconnection syndrome? Neuropsychol Rev 13:79-92

7. Scheltens P, Leys D, Barkhof F et al (1992) Atrophy of medial temporal lobes on MRI in "probable" Alzheimer's disease and normal ageing: diagnostic value and neuropsychological correlates. J Neurol Neurosurg Psychiatry 55:967-972

8. Jack CR Jr, Barkhof F, Bernstein MA et al (2011) Steps to standardization and validation of hippocampal volumetry as a biomarker in clinical trials and diagnostic criterion for Alzheimer's disease. Alzheimer Dement 7(474-485):e4

9. Nobili F, Salmaso D, Morbelli S et al (2008) Principal component analysis of FDG PET in amnestic MCI. Eur J Nucl Med Mol Imaging. 35:2191-2202

10. Buckner RL, Snyder AZ, Sanders AL, Raichle ME, Morris JC (2000) Functional brain imaging of young, nondemented, and demented older adults. J Cogn Neurosci 12(Suppl. 2):24-34

11. Rémy F, Mirrashed F, Campbell B, Richter W (2005) Verbal episodic memory impairment in Alzheimer's disease: a combined structural and functional MRI study. Neuroimage 25:253-266

12. Grady CL, Furey ML, Pietrini P, Horwitz B, Rapoport SI (2001) Altered brain functional connectivity and impaired short-term memory in Alzheimer's disease. Brain 124:739-756

13. Raichle ME, MacLeod AM, Snyder AZ, Powers WJ, Gusnard DA, Shulman GL (2001) A default mode of brain function. Proc Natl Acad Sci USA 98:676-682

14. Drzezga A, Becker JA, Van Dijk KR et al (2011) Neuronal dysfunction and disconnection of cortical hubs in non-demented subjects with elevated amyloid burden. Brain 134:1635-1646

15. Liu Y, Wang K, Yu C et al (2008) Regional homogeneity, functional connectivity and imaging markers of Alzheimer's disease: a review of resting-state fMRI studies. Neuropsychologia 46:1648-1656

16. Devanand DP, Habeck CG, Tabert MH et al (2006) PET network abnormalities and cognitive decline in patients with mild cognitive impairment. Neuropsychopharmacology 31:1327-1334

17. Sheline YI, Raichle ME, Snyder AZ et al (2010) Amyloid plaques disrupt resting state default mode network connectivity in cognitively normal elderly. Biol Psychiatry 67:584-587

18. Horwitz B, Duara R, Rapoport SI (1984) Intercorrelations of glucose metabolic rates between brain regions: application to healthy males in a state of reduced sensory input. J Cereb Blood Flow Metab 4:484-499

19. Horwitz B, Grady CL, Schlageter NL, Duara R, Rapoport SI (1987) Intercorrelations of regional cerebral glucose metabolic rates in Alzheimer's disease. Brain Res 407:294-306

20. Mosconi L, Pupi A, De Cristofaro MT, Fayyaz M, Sorbi S, Herholz K (2004) Functional interactions of the entorhinal cortex: an 18F-FDG PET study on normal aging and Alzheimer's disease. J Nucl Med 45:382-392

21. Lee DS, Kang H, Kim H et al (2008) Metabolic connectivity by interregional correlation analysis using statistical parametric mapping (SPM) and FDG brain PET; methodological development and patterns of metabolic connectivity in adults. Eur J Nucl Med Mol Imaging 35:1681-1691
22. Morbelli S, Drzezga A, Perneczky R et al (2012) Resting metabolic connectivity in prodromal Alzheimer's disease. A European Alzheimer Disease Consortium (EADC) project. Neurobiol Aging 33:2533-2550

23. Herholz K (2003) PET studies in dementia. Ann Nucl Med 17:79-89

24. Phelps ME, Schelbert HR, Mazziotta JC (1983) Positron computed tomography for studies of myocardial and cerebral function. Ann Intern Med 98:339-359

25. Wermke M, Sorg C, Wohlschläger AM, Drzezga A (2008) A new integrative model of cerebral activation, deactivation and default mode function in Alzheimer's disease. Eur J Nucl Med Mol Imaging 35(Suppl 1):S12-S24

26. Magistretti PJ (2000) Cellular bases of functional brain imaging: insights from neuron-glia metabolic coupling. Brain Res 886:108-112

27. Sestini S, Castagnoli A, Mansi L (2010) The new FDG brain revolution: the neurovascular unit and the default network. Eur J Nucl Med Mol Imaging 37:913-916

28. Dubois B, Feldman HH, Jacova C et al (2007) Research criteria for the diagnosis of Alzheimer's disease: revising the NINCDSADRDA criteria. Lancet Neurol 6:734-746

29. Morbelli S, Piccardo A, Villavecchia G et al (2010) Mapping brain morphological and functional conversion patterns in amnestic MCI: a voxel-based MRI and FDG-PET study. Eur J Nucl Med Mol Imaging 37:36-45

30. Di Chiro G, DeLaPaz RL, Brooks RA et al (1982) Glucose utilization of cerebral gliomas measured by $[18 \mathrm{~F}]$ fluorodeoxyglucose and positron emission tomography. Neurology 32:1323-1329

31. Johansen-Berg H, Behrens TE, Robson MD et al (2004) Changes in connectivity profiles define functionally distinct regions in human medial frontal cortex. Proc Natl Acad Sci USA 101:13335-13340

32. Moeller JR, Strother SC, Sidtis JJ et al (1987) Scaled subprofile model: a statistical approach to the analysis of functional patterns in positron emission tomographic data. J Cereb Blood Flow Metab 7:649-658

33. Azari NP, Rapoport SI, Grady CL et al (1992) Patterns of interregional correlations of cerebral glucose metabolic rates in patients with dementia of the Alzheimer type. Neurodegeneration 1:45-54

34. Lee JS, Lee DS (2005) Analysis of functional brain images using population-based probabilistic atlas. Curr Med Imaging Rev 1:81-87

35. Lee JS, Lee DS, Kim YK et al (2004) Quantification of brain images using Korean standard templates and structural and cytoarchitectonic probabilistic maps. Korean J Nucl Med 38:241-252

36. Amunts K, Zilles K (2001) Advances in cytoarchitectonic mapping of the human cerebral cortex. Neuroimaging Clin $\mathrm{N}$ Am 11:151-169

37. Huang S, Li J, Sun L, Alzheimer's disease NeuroImaging Initiative et al (2010) Learning brain connectivity of Alzheimer's disease by sparse inverse covariance estimation. Neuroimage 50:935-949

38. Mosconi L (2005) Brain glucose metabolism in the early and specific diagnosis of Alzheimer's disease. FDG-PET studies in MCI and AD. Eur J Nucl Med Mol Imaging 32:486-510

39. Meguro K, Blaizot X, Kondoh Y, Le Mestric C, Baron JC, Chavoix C (1999) Neocortical and hippocampal glucose hypometabolism following neurotoxic lesions of the entorhinal and perirhinal cortices in the non-human primate as shown by PET. Implications for Alzheimer's disease. Brain 122:1519-1531

40. Greicius MD, Srivastava G, Reiss AL, Menon V (2004) Defaultmode network activity distinguishes Alzheimer's disease from healthy aging: evidence from functional MRI. Proc Natl Acad Sci USA 101:4637-4642 
41. Dickerson BC, Sperling RA (2009) Large-scale functional brain network abnormalities in Alzheimer's disease: insights from functional neuroimaging. Behav Neurol 21:63-75

42. Morbelli S, Perneczky R, Drzezga A et al (2013) Metabolic networks underlying cognitive reserve in prodromal Alzheimer disease: a European Alzheimer disease consortium project. J Nucl Med 54:894-902

43. Persson J, Lustig C, Nelson JK, Reuter-Lorenz PA (2007) Age differences in deactivation: a link to cognitive control? J Cogn Neurosci 19:1021-1032

44. Bosch B, Bartrés-Faz D, Rami L et al (2010) Cognitive reserve modulates task-induced activations and deactivations in healthy elders, amnestic mild cognitive impairment and mild Alzheimer's disease. Cortex 46:451-461

45. Stern Y (2002) What is cognitive reserve? Theory and research application of the reserve concept. J Int Neuropsychol Soc 8:448-460

46. Garibotto V, Borroni B, Kalbe E et al (2008) Education and occupation as proxies for reserve in aMCI converters and AD: FDG-PET evidence. Neurology 71:1342-1349
47. Alexander GE, Furey ML, Grady CL et al (1997) Association of premorbid intellectual function with cerebral metabolism in Alzheimer's disease: implications for the cognitive reserve hypothesis. Am J Psychiatry 154:165-172

48. Scarmeas N, Zarahn E, Anderson KE et al (2003) Cognitive reserve modulates functional brain responses during memory tasks: a PET study in healthy young and elderly subjects. Neuroimage 19:1215-1227

49. Stern Y, Habeck C, Moeller J et al (2005) Brain networks associated with cognitive reserve in healthy young and old adults. Cereb Cortex 15:394-402

50. Toussaint PJ, Perlbarg V, Bellec P et al (2012) Resting state FDG-PET functional connectivity as an early biomarker of Alzheimer's disease using conjoint univariate and independent component analyses. Neuroimage 63:936-946 